\title{
Temporomandibular Disorders and Oral Features in Systemic Lupus Erythematosus Patients: An Observational Study of Symptoms and Signs
}

\author{
Vito Crincoli1 ${ }^{\circledR}$, Maria Grazia Piancino ${ }^{2}$, Florenzo Iannone ${ }^{3}$, Mariella Errede ${ }^{1}$, Mariasevera Di Comite ${ }^{1}$ \\ 1. Department of Basic Medical Sciences, Neurosciences and Sensory Organs, "Aldo Moro" University of Bari, Italy. \\ 2. Department of Surgical Sciences, University of Turin, Italy. \\ 3. Department of Emergency and Organ Transplantation, "Aldo Moro" University of Bari, Italy. \\ $\square$ Corresponding author: Prof. Vito Crincoli, Department of Basic Medical Sciences, Neurosciences and Sensory Organs, Piazza Giulio Cesare 11, 70124, Bari,
} Italy. Phone: 00390805478883; Fax: 00390805478743; e-mail: vito.crincoli@uniba.it

(C) The author(s). This is an open access article distributed under the terms of the Creative Commons Attribution License (https://creativecommons.org/licenses/by/4.0/). See http:/ /ivyspring.com/terms for full terms and conditions.

Received: 2019.07.31; Accepted: 2019.10.29; Published: 2020.01.01

\begin{abstract}
Aims: Systemic Lupus Erythematosus (SLE) is a connective tissue disease characterized by a wide range of pleomorphic pictures, including mucocutaneous, renal, musculoskeletal and neurological symptoms. It involves oral tissues, with hyposalivation, tooth decay, gingivitis, angular cheilitis, ulcers and glossitis. Temporomandibular disorders represent a heterogeneous group of inflammatory or degenerative diseases of the stomatognatic system, with algic and/or dysfunctional clinical features involving temporomandibular joint (TMJ) and related masticatory muscles. The aim of this study was to investigate the prevalence of oral manifestations and temporomandibular disorders (TMD) in SLE patients (LP) compared with a control group.

Methods: Fifty-five patients ( 9 men and 46 women) with diagnosed Lupus were recruited in the study group. A randomly selected group of 55 patients, matched by sex and age, served as control group. The examination for TMD symptoms and signs was based on the standardized Research Diagnostic Criteria for Temporomandibular Disorders (RDC/TMD) through a questionnaire and clinical examination.

Results: Lupus patients complained more frequently (95.8\%) of oral and TMJ symptoms (dysgeusia, stomatodynia, masticatory muscle pain during function, neck and shoulder muscles pain and presence of tinnitus) but only xerostomia $\left(X^{2}=4,1548 p=0,0415\right)$, temple headache $\left(X^{2}=4,4542 p=0,035\right)$ and the sensation of a stuck jaw (Mid-p-test $p=0,043)$ were significant. About signs, cheilitis $(p=0,0284)$ oral ulcers $\left(X^{2}=4,0104\right.$ $p=0,045$ ) and fissured tongue are significantly more frequent in study group. The salivary flow was significantly decreased in the study group respect to the control one $(p<0.0001)$. As regard to the oral kinematics, restricted movements (RM) in protrusion and left lateral movement were significantly different between study group and controls. In particular, $85,2 \%$ of Lp showed limited protrusion versus $56,4 \%$ of controls $\left(X^{2}=10,91\right.$ $p<0,001) ; 59,3 \%$ of $L_{p}$ had also a limitation during left lateral movement versus $47,3 \%$ of controls $(T=2,225$ $p=0,0282$ ). About bruxism, only the indentations on the lateral edges of the tongue were found in Lp group $(72,7 \%)$, with a significant difference respect to controls $\left(X^{2=7,37} p=0,007\right)$.

Conclusions: While masticatory muscles have an overlapping behavior in both groups, the findings collected show a more severe TMJ kinematic impairment in Lp than in controls, with protrusion and left lateral movements significantly different. In addition, a remarkable reduction of salivary flow has been detected in Lp compared to controls. In conclusion, this autoimmune disease seems to play a role in oral manifestations and TMJ disorders, causing an increase in orofacial pain and an altered chewing function.
\end{abstract}

Key words: Systemic Lupus Erythematosus, oral features, temporomandibular disorders, RDC/TMD

\section{Introduction}

Lupus Erythematosus (LE) is a connective tissue disease and can be classified into two major forms: the systemic (SLE) and cutaneous (CLE) one. The former is a chronic, multisystem, rheumatic disorder, present at any age, with an etiology still unknown. It has a wide range of pleomorphic pictures, including muco- 
cutaneous, renal, musculoskeletal and neurological symptoms.

The latter is further divided into three subtypes: acute (ACLE), subacute (SCLE) and chronic (CCLE) [1]. The discoid LE (DLE) is the most common variant of CCLE, characterized by erythematosus macules and plaques, follicular occlusion, desquamation, telangiectasia and atrophy. The two categories can occur together or separately [2].

Annual estimated incidence of SLE from 1970s to $2000 \mathrm{~s}$ is about $1-10 / 100.000$ and the prevalence is about 5.8-13/100.000. Cutaneous forms are considered 2-3 times more frequent than systemic one [3]. Women between 15 and 40 years are more frequently affected than men, with a preponderance of 6 up 10:1 [4,5]. Although Juvenile SLE (JSLE) is rarer, children usually experience higher disease activity and a more aggressive course compared to adults [6].

The mortality rate associated with the condition is around 3-5 times higher than that observed in the general population and is mostly attributed to the chronic inflammatory processes [7].

Pathogenesis of LE is multifactorial, involving genetic and environmental triggers [5]. About genetic factors, population studies reveal a relationship between the susceptibility to LE and human leukocyte antigens (HLA) class II and class III genes polymorphism. In patients with the expression of HLA DR2 and DR3, SLE autoantibodies are produced: (i) extractable nuclear antigens or ENA, such as anti-Ro, anti-Sm, anti-La, anti-nRNP; (ii) antinuclear antibodies or ANA, such as anti-dsDNA and anti-ssDNA. Moreover, patients with complement component $\mathrm{C} 1 \mathrm{q}$ deficit have the highest risk to develop LE, because of a reduced clearance of apoptotic bodies.

Also sex hormones and a defective hypothalamus-pituitary-adrenal (HPA) axis influence the susceptibility and the progression of the disease. In fact, increased estrogenic and reduced androgenic hormonal activity has been demonstrated in SLE patients (Lp) of both sex. Moreover, HPA axis is involved in the stress system, by increasing glucocorticoids, which results essential for prevention of dysregulated autoimmune answer. Environmental factors include infectious agents, diet, toxins/drugs, physical/chemical agents, hair dyes and tobacco smoke [8].

Clinically the disease has a variable course and a relapsing-remitting trend [4]. Several organs and systems are affected (muscles, skeleton, joints, lungs, kidneys, skin, blood vessels, nervous system), so the pathology is characterized by different manifestations. The most frequent and characteristic feature is the malar rash ("butterfly" rash), associated with arthritis, glomerulonephritis, psychosis and seizure, serosity, fever, fatigue, weight loss, anemia [9]. Mucocutaneous involvement includes alopecia, discoid lesions, photosensitivity and oral lesions [4]. These are red macules or plaques, often localized on the hard palate, leukoplakic plaques, typical of buccal mucosa, lichenoid lesions, and ulcers on lips.

Hyposalivation predispose patients with SLE to an increased risk of developing caries, gingivitis and periodontal disease, fungal infections, especially with Candida species, exfoliative or angular cheilitis and glossitis, with hyperemic and smooth tongue because of loss of papillae. A high prevalence of oral complaints such as dysphagia, dysgeusia, and glossodynia is also present, while trigeminal neuralgia is rare $[10,11,12,13,14,15,1617,18]$.

Temporomandibular disorders (TMD) is a generic term referred to clinical conditions involving the jaw muscles and temporomandibular joint (TMJ). Such disorders are related to stress, age, gender, malocclusion and other systemic factors. It is estimated that about one third of adults suffer from TMD symptoms (TMDs) [19,20,21].

TMJ can be affected in patients with SLE as they have changes in the condyles, including flattening, cortical erosions, osteophytes, sub cortical cysts, sclerosis and gradual decrease in joint space due to granulation. This involvement can be linked to disease activity, leading to a breakdown of the cartilage matrix and bone destruction. Myopathies, with reduced masticatory muscle strength and atrophy, may be part of the disease condition or associated with a long term use of corticosteroid therapy $[22,23,24,25]$.

Nevertheless, the literature lacks studies about TMJ and masticatory muscles involvement in patients with SLE, so the relationship between this disease and the temporomandibular disorders is unclear. Given this background, the aim of this study was to investigate clinically, through signs and symptoms, the prevalence of oral manifestations and temporomandibular disorders in Lp on drug therapy compared with a control group (CG), thus giving a complete survey of facial involvement in course of LES. The null hypothesis in this research was that Lp presented no differences in clinical characteristics and functional disabilities compared to a control group.

\section{Materials and Methods}

This observational study was conducted from January 2016 to February 2019 at the School of Dentistry and the Department of Rheumatology, University of Bari, Italy, in accordance with the provisions of the Declaration of Helsinki. Ethical 
approval and informed consent were obtained from each human subject.

Fifty-five patients (9 men and 46 women) with diagnosed Lupus (Lp) were recruited in the study group.

Inclusion criteria were: age $>18$ years and Caucasian ethnic origin. Exclusion criteria were: traumatic diseases, head, oral or neck neoplasia, past or present chemotherapy and radiotherapy, neurological disorders, maxillofacial treatments, past or present orthodontic treatment. Patients with other rheumatic diseases were excluded from the study. A control group (CG) of 55 subjects, matched by sex with the study group and with no immune disease history, was randomly chosen among those presenting at the Dental Clinic. Patients age ranged between 18 and 85 years, with a mean age of 44,44 (SD $=15,04)$ years in the Lp group and 46 years $(\mathrm{SD}=$ $13,49)$ in the CG.

TMD signs and symptoms were valued following the standardized Research Diagnostic Criteria for Temporomandibular Disorders (RDC/TMD) [19].

A single experienced practitioner assessed TMD and orofacial manifestations through an anamnestic questionnaire and a clinical examination.

\section{Patients history}

\section{Oral symptoms}

Through a questionnaire, patients listed the presence/absence of the following disorders:

(i)Xerostomia: characterized by dry mouth and associated to discomfort especially when they eat, speak, swallow and wear dentures. Less stringy and foamy saliva is produced [12].

(ii)Dysgeusia: defined a distorted gustatory perception. Patients report that they perceive bitter, sour, or metallic flavours [13].

(iii)Stomatodynia: a burning sensation in the mouth, associated with hurtful sensation or pain, even though oral mucosa appears clinically normal $[14,15]$.

\section{TMD symptoms}

TMDs: they include muscle pain, neck and upper shoulders stiffness, pain at masticatory muscles during mandibular functions, arthralgia (tenderness or pain in TMJ area), a feeling of locked jaw, headaches, especially at the temples $[16,19]$. Dizziness, earache and tinnitus are other less common problems that these patients also complained [26,27]. Patients described how much the disease was serious according their perception by using VAS scale (from slight tenderness to unbearable pain) and if they had a periodical or continuous symptomatology since the disease was diagnosed.
Myofascial pain (MP): while palpation does not elicit sensations of tenderness or pain in healthy muscles, ache may be provoked by compression of contract or inflamed muscles. The following masticatory muscles were palpated bilaterally: anterior, medial and posterior temporalis muscles, masseter muscle, medial pterygoid muscle, lateral pterygoid muscle with its superior and inferior head, digastric (anterior and posterior belly) muscle and mylohyoid muscles. Palpation was performed applying soft but firm pressure to the muscle mainly with the palmar surface of the thumb and of the index finger.

\section{Clinical examination}

\section{Lupus oral signs}

Oral ulcers: presence of oval or roundish sores inside the mouth.

Petechiae: red or brown pinpoint lesions not blanching on pressure, localized more frequently on the hard and soft palate [28].

Erythema: reddish and inflamed area on buccal mucosa.

Fissured tongue: anatomical condition of the tongue surface, usually asymptomatic. Grooves are distributed across the dorsal surface of the tongue and can vary in size and depth.

Cheilitis of lower lip: it is an inflammation state, characterized by redness, swelling and ulcers on lower lip.

Hyposalivation: the test was conducted asking the patient to spit saliva accumulated in the floor of the mouth without stimulation in a graduated tube every 60 seconds. The collection period lasted 5 minutes [29].

Other oral characteristics analyzed were the integrity of the dental arches or presence of partial or total edentulism, the presence/absence of prostheses (mobile, fixed or both).

\section{TMJ signs}

TMJ sounds (TMJs): they were appreciated by palpation on each side separately on mandible movement. They can be classified in: (i) clicking, (ii) crepitation. Clicking is defined as a single, clear joint sound of short duration. Crepitation is a sound similar to a rough multiple, gravel-like sound [20].

Bruxism (BRUX): it is a stereotypical jaw movement, characterized by clenching and gnashing of the teeth, during sleep or when awake [30]. By time, flattening of the dental cusps or dental mobility can occur. Bruxism is considered pathological when it causes myalgia (due to a prolonged vasoconstriction and to accumulation of catabolites in the muscle tissue) and joint pain [31]. 
Opening derangement (OD): in a healthy condition, the mandible-opening path (observing the lower midline) is straight. Alterations of the opening trajectory are:

(i) deviation: any shift of the jaw midline during opening that disappears with continued opening (a return to midline);

(ii) deflection: any shift of the midline to one side that increases with opening and persists at maximum opening [32].

\section{Restricted movements (RM)}

They are classified as:

(i) reduced opening: in a healthy system, the mouth opens by between 53 and $58 \mathrm{~mm}$. Taking into account overbite [33], a restricted mandibular opening is considered to be any distance of $<40 \mathrm{~mm}$;

(ii) reduced right and left lateral excursions, measured from upper to lower midline, when the distance is $<8 \mathrm{~mm}$;

(iii) a mandibular advancement: it is considered reduced when $<7 \mathrm{~mm}[34,35]$.

\section{Statistical analysis}

Continuous data were presented as mean and Standard Deviation (SD) and the comparisons between $\mathrm{Lp}$ and controls were assessed by means of Student's $\mathrm{T}$ test for unpaired samples. Categorical data were expressed as number and percentage and Chi-squared (with Mantel-Haenszel or Yates' corrections). Mid-P or Fisher Exact Tests were employed to compare two groups. A two-tailed $p$ value $\leq 0.05$ was considered as statistically significant. Statistical analyses were performed using Prism (GraphPad software, version 6.0, San Diego, California).

\section{Results}

\section{Characteristics of Lupus patients (Lp) and controls}

The prevalent form of disease was the systemic one (SLE), found in the $90,9 \%$ of Lp. The age at diagnosis varied between 12 and 84 years (mean $=32,9$ years, $\mathrm{SD}=16,07$ ) with a disease mean duration of 8,04 years $(\mathrm{SD}=8) ; 43,6 \%$ of patients had the pathology for less than 5 years.

The two groups, matched for age and sex, resulted similar for sociodemographic aspects, except for educational degree $\left(\chi^{2}=9,4184 p=0,0242\right)$ and occupation $\left(\chi^{2}=21,6894 p=0.0014\right)$. About education, high school degree was prominent in both groups, but graduates were more than double among controls. As regard to occupation, among Lp group housewives and jobless prevailed, while among controls public employees were prominent (Table 1).
Clinical characteristics of Lp patients and controls are reported in Table 2. The principal drugs for Lp patients and controls are reported in Table 3.

Table 1. Sociodemographic characteristics of Lupus patients (LP) and controls.

\begin{tabular}{|c|c|c|c|c|}
\hline $\begin{array}{l}\text { Sociodemographic } \\
\text { characteristics }\end{array}$ & Lp & Controls & Test & $p$ Value \\
\hline Age, mean $\pm S D$ & $44,44 \pm 15,04$ & $46 \pm 13,49$ & & \\
\hline \multicolumn{5}{|l|}{ Sex, $n(\%)$} \\
\hline Male & $9(16,4 \%)$ & $9(16,4 \%)$ & & \\
\hline Female & $46(83,6 \%)$ & $46(83,6 \%)$ & & \\
\hline \multicolumn{3}{|c|}{ Educational degree, $n(\%)$} & \multirow[t]{5}{*}{$\chi^{2}=9,4184$} & \multirow[t]{5}{*}{0,0242} \\
\hline Primary & $8(14,5 \%)$ & $5(9,1 \%)$ & & \\
\hline Secondary & $17(30,9 \%)$ & $7(12,7 \%)$ & & \\
\hline High & $25(45,5 \%)$ & $29(52,7 \%)$ & & \\
\hline Academic & $5(9,1 \%)$ & $14(25,5 \%)$ & & \\
\hline \multicolumn{2}{|l|}{ Occupation, $n(\%)$} & & \multirow[t]{7}{*}{$X^{2}=21,6894$} & \multirow[t]{7}{*}{0,0014} \\
\hline Housewife & $18(32,7 \%)$ & $13(23,6 \%)$ & & \\
\hline Retired & $5(9,1 \%)$ & $5(9,1 \%)$ & & \\
\hline Office Worker & $12(21,8 \%)$ & $11(20 \%)$ & & \\
\hline Self Employed & $3(5,5 \%)$ & $4(7,3 \%)$ & & \\
\hline Not Employed & $12(21,8 \%)$ & $1(1,8 \%)$ & & \\
\hline Public Employee & $2(3,6 \%)$ & $16(29,1 \%)$ & & \\
\hline \multicolumn{2}{|l|}{ Marital status, $n(\%)$} & & \multirow[t]{5}{*}{$\chi^{2}=7,2039$} & \multirow[t]{5}{*}{0,0657} \\
\hline Married & $29(52,7 \%)$ & $39(70,9 \%)$ & & \\
\hline Widower & $1(1,8 \%)$ & $2(3,6 \%)$ & & \\
\hline Single & $21(38,2 \%)$ & $14(25,5 \%)$ & & \\
\hline Divorced & $4(7,3 \%)$ & 0 & & \\
\hline
\end{tabular}

Table 2. Comorbidity in Lp and controls.

\begin{tabular}{lllll}
$\begin{array}{l}\text { Concomitant } \\
\text { diseases }\end{array}$ & $\mathbf{L p}, \boldsymbol{n ( \% )}$ & $\begin{array}{l}\text { Controls } n \\
\mathbf{( \% )}\end{array}$ & Test & $\begin{array}{l}\boldsymbol{p} \\
\text { Value }\end{array}$ \\
\hline Cardiopathy & $9(16,4 \%)$ & $2(3,6 \%)$ & $\chi^{2}=4,9045$ & 0,027 \\
Diabetes mellitus & $2(3,6 \%)$ & 0 & Fisher exact test & 0,248 \\
Esophageal disease & $8(14,5 \%)$ & $3(5,5 \%)$ & $\chi^{2}=2,5253$ & 0,112 \\
Gastritis & $2(3,6 \%)$ & 0 & Fisher exact test & 0,248 \\
Hypertension & $12(21,8 \%)$ & $6(10,9 \%)$ & $\chi^{2}=2,3913$ & 0,122 \\
Hypovitaminosis D & $6(10,9 \%)$ & 0 & Fisher exact test & 0,016 \\
Lung disease & $14(25,5 \%)$ & 0 & $\chi^{2}=13,8318$ & $<0,001$ \\
Osteoporosis & $10(18,2 \%)$ & $1(1,8 \%)$ & $\chi^{2}=6,4646$ & 0,011 \\
Presence of & $4(7,3 \%)$ & $6(10,9 \%)$ & $\chi^{2}=0,4400$ & 0,507 \\
removable prosthesis & & & & \\
Raynaud syndrome & $10(18,2 \%)$ & 0 & $\chi^{2}=8,9100$ & 0,003 \\
Thyroid disease & $12(21,8 \%)$ & $5(9,1 \%)$ & Mid-p-test & 0,036 \\
Renal disease & $26(47,3 \%)$ & 0 & $\chi^{2}=31,4789$ & $<0,001$ \\
Blood disease & $24(43,6 \%)$ & 0 & $\chi^{2}=28,1928$ & $<0,001$ \\
Neurological disease & $7(12,7 \%)$ & 0 & Fisher exact test & 0,006 \\
\hline
\end{tabular}

Table 3. Lp and controls' drugs.

\begin{tabular}{lllll}
\hline Drugs & Lp, $\boldsymbol{n} \mathbf{( \% )}$ & $\begin{array}{l}\text { Controls } \boldsymbol{n} \\
\mathbf{( \% )}\end{array}$ & Test & $\boldsymbol{p}$ Value \\
\hline Azathioprine & $8(14,5 \%)$ & 0 & Fisher exact test & 0,003 \\
Belimumab & $2(3,6 \%)$ & 0 & Fisher exact test & 0,248 \\
Cyclophosphamide & $3(5,5 \%)$ & 0 & Fisher exact test & 0,122 \\
Cyclosporine & $6(10,9 \%)$ & 0 & Fisher exact test & 0,0135 \\
Corticosteroids & $41(74,5 \%)$ & 0 & Fisher exact test & $<0,001$ \\
Hydroxychloroquine & $31(56,4 \%)$ & 0 & Fisher exact test & $<0,001$ \\
Leflunomide & $1(1,8 \%)$ & 0 & Fisher exact test & 0,500 \\
Methotrexate & $4(7,3 \%)$ & 0 & Fisher exact test & 0,059 \\
Mycophenolate Mofetil & $15(27,3 \%)$ & 0 & Fisher exact test & $<0,001$ \\
Rituximab & $2(3,6 \%)$ & 0 & Fisher exact test & 0,248 \\
\hline
\end{tabular}


Table 4. Subjective complaints of oral discomfort in $L p$ and controls.

\begin{tabular}{lllll}
\hline Oral symptoms & $\mathbf{L p}, \boldsymbol{n} \mathbf{( \% )}$ & Controls, $\boldsymbol{n} \mathbf{( \% )}$ & $\chi^{2}$ & $\boldsymbol{p}$ Value \\
\hline Xerostomia & $17(30,9 \%)$ & $8(14,5 \%)$ & 4,1548 & 0,0415 \\
Dysgeusia & $5(9,1 \%)$ & $2(3,6 \%)$ & 1,3731 & 0,241 \\
Stomatodynia & $4(7,3 \%)$ & $3(5,5 \%)$ & 0,1526 & 0,696 \\
\hline
\end{tabular}

Table 5. TMD symptoms in Lp and controls.

\begin{tabular}{lllll}
\hline TMDs & Lp, $\boldsymbol{n} \mathbf{( \% )}$ & Controls, $\boldsymbol{n} \mathbf{( \% )}$ & Test & $\boldsymbol{p}$ Value \\
\hline Arthralgia & $17(30,9 \%)$ & $10(18,2 \%)$ & $\chi^{2}=2,4052$ & 0,121 \\
$\begin{array}{l}\text { Temple headache } \\
\text { Sensation of stuck jaw }\end{array}$ & $29(52,7 \%)$ & $18(32,7 \%)$ & $\chi^{2}=4,4542$ & 0,035 \\
$\begin{array}{l}\text { Masticatory muscle } \\
\text { pain during function }\end{array}$ & $14(25,5 \%)$ & $6(10,9 \%)$ & Mid-p-test & 0,043 \\
$\begin{array}{l}\text { Neck and shoulder } \\
\text { muscles pain }\end{array}$ & $38(69,1 \%)$ & $35(63,6 \%)$ & $\chi^{2}=0,3665$ & 0,545 \\
tinnitus & $16(29,1 \%)$ & $10(18,1 \%)$ & $\chi^{2}=1,8132$ & 0,178 \\
\hline
\end{tabular}

Table 6. Myofascial pain in Lp and controls.

\begin{tabular}{lclll}
\hline Muscle & Lp, $\boldsymbol{n}(\%)$ & $\begin{array}{l}\text { Controls, } \\
\boldsymbol{n}(\%)\end{array}$ & Test & $\begin{array}{l}\boldsymbol{p} \\
\text { Value }\end{array}$ \\
\hline Anterior temporalis muscles & $21(38,2 \%)$ & $17(30,9 \%)$ & $\chi^{2}=0,6433$ & 0,423 \\
Medial temporalis muscles & $16(29,1 \%)$ & $12(21,8 \%)$ & $\chi^{2}=0,7666$ & 0,381 \\
Posterior temporalis muscles & $11(20,0 \%)$ & $10(18,2 \%)$ & $\chi^{2}=0,0589$ & 0,808 \\
Superficial masseter muscles & $29(52,7 \%)$ & $25(45,5 \%)$ & $\chi^{2}=0,5820$ & 0,446 \\
Deep masseter muscles & $29(52,7 \%)$ & $26(47,3 \%)$ & $\chi^{2}=0,3273$ & 0,567 \\
medial pterygoid muscles & $29(52,7 \%)$ & $30(54,5 \%)$ & $\chi^{2=}=0,0366$ & 0,848 \\
lateral pterygoid muscle & $30(54,5 \%)$ & $37(67,3 \%)$ & $\chi^{2=1,8709}$ & 0,171 \\
digastric muscle - anterior belly & $10(18,2 \%)$ & $11(20,0 \%)$ & $\chi^{2}=1,0589$ & 0,808 \\
digastric muscle - posterior belly & $13(23,6 \%)$ & $18(14,5 \%)$ & $\chi^{2}=1,4714$ & 0,225 \\
Mylohyoid muscles & $14(25,5 \%)$ & $11(20,0 \%)$ & $\chi^{2}=0,4659$ & 0,495 \\
\hline
\end{tabular}

Table 7. Oral signs for Lp and controls.

\begin{tabular}{lllll}
\hline Oral signs & Lp, $\boldsymbol{n} \mathbf{( \% )})$ & $\begin{array}{l}\text { Controls, } \boldsymbol{n} \\
\mathbf{( \% )}\end{array}$ & Test & $\begin{array}{l}\boldsymbol{p} \\
\text { Value }\end{array}$ \\
\hline Candidiasis & $1(1,8 \%)$ & 0 & Fisher exact test & 0,500 \\
Cheilitis & $5(9,1 \%)$ & 0 & Fisher exact test & 0,0284 \\
Erythema & $3(5,5 \%)$ & 0 & Fisher exact test & 0,122 \\
Petechiae & $3(5,5 \%)$ & 0 & Fisher exact test & 0,122 \\
Fissured tongue & $7(12,7 \%)$ & 0 & Fisher exact test & 0,006 \\
Oral ulcers & $11(20 \%)$ & $3(5,5 \%)$ & $\chi^{2}=4,0104$ & 0,045 \\
Erythematous and & $2(3,6 \%)$ & 0 & Fisher exact test & 0,248 \\
hyperkeratotic areas & & & & \\
Others & $4(7,3 \%)$ & $12(21,8 \%)$ & $\chi^{2}=4,6383$ & 0,031 \\
\hline
\end{tabular}

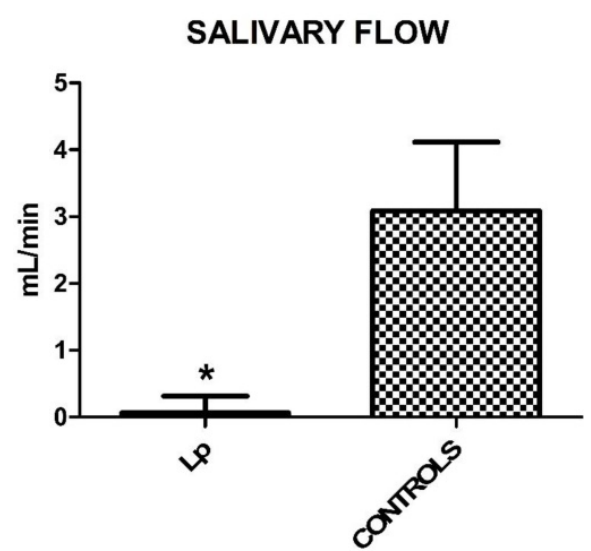

Figure 1. Mean values $\pm S D$ of $L p$ and control group. Lp patients show a reduced salivary flow.

\section{Lupus oral symptoms}

Twenty-nine Lp $(52,7 \%)$ complained of one or more oral symptoms compared to 24 of controls $(43,6 \%)$. A statistically significant difference between two groups was found only for xerostomia (Table 4).

\section{TMD symptoms}

The assessment of TMDs showed that $94,5 \% \mathrm{Lp}$ and $90,9 \%$ of controls complained one or more symptoms. Statistically significant differences weren't found between the two whole groups $\left(\chi^{2}=0,5392\right.$ $p=0,463$ ).

Arthralgia, temple headache, sensation of a stuck jaw, masticatory muscle pain during function, neck and shoulder muscles pain and presence of tinnitus are more frequent in $\mathrm{Lp}$ than in controls, but only temple headache $\left(\chi^{2}=4,4542 p=0,035\right)$ and the difficulty in opening mouth (Mid-p-test $p=0,043$ ) are significant (Table 5).

Myofascial pain (MP) evoked by palpation was detected in 70,9\% Lp and 76,4\% of controls, revealing a similar frequency between $\mathrm{Lp}$ and controls $\left(\chi^{2}=0,4215 p=0,516\right)$. Data collected for each muscle couple are reported in Table 6 .

\section{Lupus oral signs}

At the clinical examination, 52,7\% of Lp have at least one oral sign respect to $43,6 \%$ of controls, but this difference is not statistically significant $\left(\chi^{2}=0.91\right.$ $p=0,340)$. However, there are statistically significant differences between the two groups for some observed signs. Cheilitis, oral ulcers and fissured tongue are more frequent in study group. Other signs (oral respiration, gingival recessions, herpes labialis, BMS) are more frequently observed in controls than $\operatorname{Lp}\left(\chi^{2}=4,6383 p=0,031\right)$. Table 7 lists the main findings collected from the oral examination.

Regarding the extent of salivary flow, measured in 5 minutes, there was a statistically significant reduction in the Lp compared to controls (Student's T Test, $p<0,0001)$. Mean values and SD are: $0.254 \pm 0.395$ for Lp and $3.197 \pm 0.571$ for control group (Figure 1).

\section{TMJ signs}

As regard to restricted movements (RM), protrusion and left lateral movement were significantly different between $\mathrm{Lp}$ and controls. In particular, $85,2 \%$ of $\mathrm{Lp}$ showed limited protrusion versus $56,4 \%$ of controls $\left(x^{2}=10,91 p<0,001\right) ; 59,3 \%$ of Lp had a limitation during left lateral movement versus $47,3 \%$ of controls ( $T=2,225 p=0,0282)$.

The prevalence of Opening derangement (OD) was higher in Lp $(45,20 \%)$ than in controls $(44,24 \%)$, although this difference was not statistically significant $\left(\chi^{2}=0,05 p=0,5347\right)$. Measurement data 
related to movements are reported in Table 8.

Bruxism was more frequent in control group than Lupus one $(38,2 \%$ versus $32,7 \%)$. No significant difference between the two groups was found for wear facets and oral frictional hyperkeratosis (morsicatio buccarum). Only the indentations on the lateral edges of the tongue were found in Lp group $(72,7 \%)$, with a significant difference respect to controls $\left(\chi^{2}=7,37 p=0,007\right)$. Table 9 lists parafunction (bruxism and clenching) signs.

Finally, joint sounds were more frequent in controls $(25,5 \%)$ than Lp $(16,4 \%)$ but with no statistically significant difference.

Table 8. Restricted Movements (RM).

\begin{tabular}{lllll}
\hline $\begin{array}{l}\text { Measurements }(\mathbf{m m}) ; \\
\text { mean } \pm \text { SD }\end{array}$ & Lp & Controls & T Student & $p$ Value \\
\hline Opening & $45,20 \pm 8,61$ & $44,24 \pm 7,65$ & 0,6228 & 0,5347 \\
Laterotrusion right & $5,85 \pm 3,49$ & $6,86 \pm 2,5$ & 1,681 & 0,0957 \\
Laterotrusion left & $6,13 \pm 4,02$ & $7,636 \pm 2,98$ & 2,225 & 0,0282 \\
Protrusion & $4,09 \pm 2,36$ & $6,091 \pm 2,75$ & 4,069 & $<0,0001$ \\
\hline
\end{tabular}

Table 9. Parafunctional signs.

\begin{tabular}{lllll}
\hline Parafunctional signs & Lp & Controls & Test & $p$ Value \\
\hline Wear facets & $32(58,2 \%)$ & $25(45,5 \%)$ & $\chi^{2}=1,7842$ & 0,550 \\
Oral frictional hyperkeratosis & $26(47,3 \%)$ & $19(34,5 \%)$ & $\chi^{2}=1,8427$ & 0,175 \\
$\begin{array}{l}\text { Indentations on lateral edges of } \\
\text { tongue }\end{array}$ & $40(72,7 \%)$ & $25(45,5 \%)$ & $\chi^{2}=7,3709$ & 0,007 \\
\hline
\end{tabular}

\section{Discussion}

The present study analyzed the prevalence of symptoms and sign of both mucosal damage and temporomandibular dysfunction in $\mathrm{Lp}$ compared with healthy controls. The present cohort is larger respect to the sample size of other studies investigating TMD and LE: 55 Lp versus 2 in Liebling and Gold [36], 2 in Simoes et al. [37], 22 in Aliko et al. [23], 25 in Aceves-Avila et al. [38], 37 in Jonsson et al. [39]. Lopez-Labady's work examined $90 \mathrm{Lp}$, but only oral manifestations were described, while TMD were omitted [1].

About sociodemographic characteristics, women (46 females and 9 males) are more affected than men, in accordance with the literature [4,5]. Jobless and housewives were most represented in Lp, while public employees were most represented among controls. About education, graduates were more than double among controls. These data could be explained with a progressive physical disability in patients with SLE. In fact, several comorbidities (Table $2)$, such as renal $\left(\chi^{2}=31,4789 \mathrm{p}<0,001\right)$, lung $\left(\chi^{2}=13,8318 p<0,001\right)$ and blood diseases $\left(\chi^{2}=28,1928\right.$ $p<0,001)$, lead to an impairment of patients' quality of life.

Mucosal symptoms and signs were evaluated in
Lp and control group: xerostomia, among symptoms, and cheilitis, fissured tongue and ulcers, among signs, show statistically significant findings. In detail, 30,9\% of Lp versus $14,5 \%$ of controls $\left(\chi^{2}=4,1548 p=0,0415\right)$ complained xerostomia, with a feeling of mouth dryness (sicca syndrome). The main cause appears to be a lymphocytic sialadenitis associated with a secondary Sjögren's syndrome. In fact, according to Brennan et al., 7,5-30\% of Lp have Sjogren syndrome, with cheilitis, erythema, hyperkeratotic areas, ulcerations, periodontal diseases, atrophic or fissured tongue, caries, candidiasis [4,10,29,40,41]. Also drugs can cause xerostomia: $74,5 \%$ of Lp take corticosteroids $(p=0,001)$ from the diagnosis of the disease, often in addition to others drugs, such as hydroxychloroquine (56,4\%), Mycophenolate Mofetil (27,3\%), azathioprine $(14,5 \%)$, cyclosporine $(10,9 \%)$. This remarkable symptom is confirmed by the salivary test, with a significantly reduced salivary flow in Lp patients. Consequently, the hyposalivation may be responsible of cheilitis $(p=0,0284)$, fissured tongue $(p=0,006)$ and oral ulcers $(p=0,045)$, statistically more frequent in Lp than controls.

A higher prevalence of TMD symptoms was detected in $\operatorname{Lp}(94,5 \%)$ compared to the respective outcomes in healthy patients $(90,9 \%)$. However, only temple headache $\left(\chi^{2}=4,4542 p=0,035\right)$ and sensation of stuck jaw (Mid-p-test $p=0,043$ ) are significant (Table 5). Myopathies, with reduced muscle strength and atrophy, may be part of the disease condition or associated with the continuous use of corticosteroid therapy. Myofascial pain (MP) evoked by palpation was detected in $70,9 \% \mathrm{Lp}$ and $76,4 \%$ of controls $\left(X^{2}=0,4215 \mathrm{p}=0,5162\right)$, so masticatory muscles show overlapping data in both groups (Table 6). The results of the present investigation are in accordance with literature. To our knowledge, in fact, in no previous examined study, pain was elicited during muscles palpation, except in Bade's study, who valuated only one patient with SLE (masseter, anterior temporal and lateral pterygoid muscles were painful) [42].

Also temporomandibular joint (TMJ) involvement can affect patients with LES, as they have changes in the condyles, including flattening, erosions, osteophytes and sclerosis. These alterations can be linked to disease activity, leading to loss of joint cartilage and bone destruction.

A valuated symptom was arthralgia (pain around TMJ area). It is more frequent in Lp $(30,9 \%)$ respect to controls $(18,2 \%)$, but it isn't statistically significant. This result is in contrast with previous studies, where pain during TMJ palpation was always found $[23,39,42]$.

In this investigation, the presence of TMJ sounds, was overall more evident in controls $(25,5 \%)$ than Lp 
(16,4\%), but not significantly different. Also Aliko [23], and Jonsson [39] did not found a significant difference in Lp and controls.

Maybe the explanation is that crepitation often indicates structural damage to the TMJ and the drugs early given in LE could have the potential to reduce or slow down joint damage [22]. Moreover, the "healthy" sample was selected from people attending at Dental Clinic, who could complain TMD more frequently than general population, and this could be a limitation of the present study.

The main expected finding was a more severe restriction on mandibular movements in Lp patients than in controls. Protrusion and left lateral movement were significantly different between the two groups. In particular, $85,2 \%$ of Lp showed limited protrusion versus $56,4 \%$ of controls $\left(\chi^{2}=10,91 p<0,001\right) ; 59,3 \%$ of Lp had a limitation during left lateral movement versus $47,3 \%$ of controls ( $\mathrm{T}=2,225 p=0,0282)$.

However, the mouth opening value (mean $=45,20$ $\pm 8,615)$ was not statistically significant respect to control group (25,5\% of Lp versus $23,6 \%$ of controls). Also Aliko showed a restricted opening only in $4,5 \%$ of patients [23], while for Jonsonn this sign is statistically significant [39].

\section{Conclusions}

The aim of this observational study was to investigate the prevalence of TMD symptoms and signs as well as oral implications in patients with SLE. While masticatory muscles have an overlapping behavior in both groups, the findings collected show a more severe TMJ kinematic impairment in Lp patients than in controls, with protrusion and left lateral movements significantly different. Also a remarkable reduction of salivary flow was detected in $\mathrm{Lp}$ compared to controls $(p<0,0001)$.

Lupus, like other autoimmune diseases, seems to play a role in oral and TMJ alterations, causing an increase in orofacial pain and an impairment of jaw mobility. An interdisciplinary collaboration between the stomatologist and the rheumatologist would be appropriate, thus giving a complete survey of facial involvement in course of LES and a more efficient treatment of this disease.

\section{Abbreviations}

LE: Lupus Erythematosus; SLE: Systemic Lupus Erythematosus; CLE: Cutaneous Lupus Erythematosus; SCLE: Subacute Cutaneous Lupus Erythematosus; ACLE: Acute Cutaneous Lupus Erythematosus; CCLE: Chronic Cutaneous Lupus Erythematosus; DLE: Discoid Lupus Erythematosus; HLA: Human Leukocyte Antigens; ENA: Extractable Nuclear Antigens; ANA: Anti Nuclear Antigens; HPA:
Hypothalamus- Pituitary- Adrenal; ANUG: Acute Necrotizing Ulcerative Gingivitis; Lp: Lupus Patients; CG: Control Group; TMJ: Temporomandibular Joint; TMD: Temporomandibular Disorders; SD: standard deviation; TMDs: Symptoms of Temporomandibular Disorders; MP: Myofascial Pain; TMJs: Sounds of Temporomandibular Joint; BRUX: bruxism; BMS: Burning Mouth Syndrome; RM: Restricted Movements; OD: Opening Derangement; RDC/TMD: Research Diagnostic Criteria for Temporomandibular Disorders; VAS: Visual Analogue Scale.

\section{Acknowledgements}

Study Design: Vito Crincoli, Maria Grazia Piancino; Data Collection: Florenzo Iannone, Mariella Errede;Statistical Analysis: Mariasevera Di Comite; Data Interpretation: Vito Crincoli, Mariasevera Di Comite, Mariella Errede; Manuscript Preparation: Vito Crincoli, Mariasevera Di Comite; Literature Search: Florenzo Iannone, Mariella Errede.

\section{Competing Interests}

The authors have declared that no competing interest exists.

\section{References}

1. López-Labady J, Villarroel-Dorrego M, González N, Pérez R, Mata de Henning M. Oral manifestations of systemic and cutaneous lupus erythematosus in a Venezuelan population. J.Oral Pathol. Med. 2007; 36: 524-7.

2. Grönhagen CM, Nyberg F. Cutaneous lupus erythematosus: An update. Indian Dermatol. Online J. 2014; 5: 7-13.

3. Jarukitsopa S, Hoganson DD, Crowson CS, Sokumbi O, Davis MD, Michet CJ Jr, Matteson EL, Maradit Kremers H, Chowdhary VR. Epidemiology of systemic lupus erythematosus and cutaneous lupus erythematosus in a predominantly white population in the United States. Arthritis Care Res. 2015; 67: 817-28

4. Brennan MT, Valerin MA, Napeñas JJ, Lockhart PB. Oral manifestations of patients with lupus erythematosus. Dent. Clin. North Am. 2005; 49: 127-41.

5. Herrmann M, Voll RE, Kalden JR. Etiopathogenesis of systemic lupus erythematosus. Immunol. Today 2000; 21: 424-6.

6. Abrão AL, Falcao DP, de Amorim RF, Bezerra AC, Pombeiro GA, Guimarães LJ, Fregni F, Silva LP, da Mota LM. Salivary proteomics: A new adjuvant approach to the early diagnosis of familial juvenile systemic lupus erythematosus. Med Hypotheses. 2016; 89: 97-100.

7. Cervera R, Khamashta MA, Font J, Sebastiani GD, Gil A, Lavilla P, Mejía JC, Aydintug AO, Chwalinska-Sadowska H, de Ramón E, Fernández-Nebro A Galeazzi M, Valen M, Mathieu A, Houssiau F, Caro N, Alba P, Ramos-Casals M, Ingelmo M, Hughes GR; European Working Party on Systemic Lupus Erythematosus. Morbidity and mortality in systemic lupus erythematosus during a 10-year period: a comparison of early and late manifestations in a cohort of 1,000 patients. Medicine (Baltimore). 2003; 82(5): 299-308.

8. Mok CC, Lau CS. Pathogenesis of Systemic Lupus Erythematosus. J. Clin. Pathol. 2003; 56: 481-90.

9. Klippel JH. Systemic Lupus Erythematosus: demographics, prognosis, and outcome. J. Rheumatol. Suppl. 1997; 48: 67-71.

10. Heath KR, Rogers RS, Fazel N. Oral manifestations of connective tissue disease and novel therapeutic approaches. Dermatol. Online J. 2015; 16: 2.

11. Gualtierotti R, Marzano AV, Spadari F, Cugno M. Main Oral Manifestations in Immune-Mediated and Inflammatory Rheumatic Diseases. J. Clin. Med. 2019; 8(1): 21.

12. Greenspan D. Xerostomia: diagnosis and management. Oncology (Williston Park). 1996; 10 (Suppl 3): S7-S11.

13. Mott AE, Grushka M, Sessle BJ. Diagnosis and management of taste disorders and burning mouth syndrome. Dent. Clin. North Am. 1993; 37: 33-71.

14. Fedele S, Fricchione G, Porter SR, Mignogna MD. Burning mouth syndrome (stomatodynia). Q.J.M. 2007; 100: 527-30.

15. de Tommaso M, Lavolpe V, Di Venere D, Corsalini M, Vecchio E, Favia G, Sardaro M, Livrea P, Nolano M. A case of unilateral burning mouth syndrome of

neuropathic origin. Headache. 2011; 51(3): 441-443. 
16. Corsalini M, Daniela DV, Biagio R, Gianluca S, Alessandra L, Francesco P. Evidence of Signs and Symptoms of Craniomandibular Disorders in Fibromyalgia Patients. Open Dent J. 2017; 11: 91-98.

17. Abrão AL, Santana CM, Bezerra AC, Amorim RF, Silva MB, Mota LM, Falcão DP. What rheumatologists should know about orofacial manifestations of autoimmune rheumatic diseases. Rev Bras Reumatol Engl Ed. 2016; 56(5): $441-450$.

18. Kumar V, Kaur J, Pothuri P, Bandagi S. Atypical Trigeminal Neuralgia: A Rare Neurological Manifestation of Systemic Lupus Erythematosus. Am J Case Rep. 2017; 18: 42-45.

19. Dworkin SF, LeResche L. Research diagnostic criteria for temporomandibular disorders: review, criteria, examinations and specifications, critique. J Craniomandib. Disord. 1992; 6: 301-55.

20. Tecco S, Crincoli, V, Di Bisceglie B, Saccucci M, Macrì M, Polimeni A, Festa F. Signs and symptoms of temporomandibular joint disorders in Caucasian children and adolescents. Cranio. 2011; 29: 71-9.

21. Crincoli V, Di Comite M, Di Bisceglie MB, Fatone L, Favia G. Temporomandibular Disorders in Psoriasis Patients with and without Psoriatic Arthritis: An Observational Study. Int. J. Med. Sci. 2015; 12 (Suppl 4): 341-8.

22. Clarke B.A., Drujan D., Willis M.S. The E3 Ligase MuRF1 degrades myosin heavy chain protein in dexamethasone-treated skeletal muscle. Cell Metab. 2007; 6: 376-385.

23. Aliko A, Ciancaglini R, Alushi A, Tafaj A, Ruci D.Temporomandibular joint involvement in rheumatoid arthritis, systemic lupus erythematosus and systemic sclerosis. Int J Oral Maxillofac Surg. 2011; 40(7):704-9.

24. Golin SL, Sinicato NA, Valle-Corotti K, Fuziy A, Nahas-Scocate AC, Appenzeller S, Costa ALF. Assessment of condyle, masseter and temporal muscles volumes in patients with juvenile systemic lupus erythematosus: A cross-sectional study. J Oral Biol Craniofac Res. 2017; 7(2):89-94.

25. Crincoli $\mathrm{V}$, Anelli MG Quercia $\mathrm{E}$ Piancino $M G$ Di Comite $\mathrm{M}$. Temporomandibular Disorders and Oral Features in Early Rheumatoid Arthritis Patients: An Observational Study. Int J Med Sci. 2019; 16(2):253-263.

26. Buergers R, Kleinjung T, Behr M, Vielsmeier V. Is there a link between tinnitus and temporomandibular disorders? J. Prosthet. Dent. 2014; 111: 222-7.

27. Solarino B, Coppola F, Di Vella G, Corsalini M, Quaranta N. Vestibular evoked myogenic potentials (VEMPs) in whiplash injury: a prospective study. Acta Otolaryngol. 2009; 129(9):976-981.

28. Scully C, Porter S. Orofacial disease: update for the dental clinical team: 4. Red, brown, black and bluish lesions. Dental update.1999; 26: 169-173.

29. Crincoli V, Di Comite M, Guerrieri M, Rotolo RP, Limongelli L, Tempesta A, Iannone F, Rinaldi A, Lapadula G, Favia G. Orofacial Manifestations and Temporomandibular Disorders of Sjögren Syndrome: An Observational Study. Int J Med Sci. 2018; 8;15(5):475-483.

30. Lavigne GJ, Khoury S, Abe S, Yamaguchi T, Raphael K. Bruxism physiology and pathology: an overview for clinicians. J. Oral Rehabil. 2008; 35: 476-94.

31. Bell WE. Orofacial pains: differential diagnosis, 2nd ed., Chicago, IL, USA: Year Book Medical Publishers Inc; 1979.

32. Sener S, Akgunlu F. Correlation between the Condyle Position and Intra-Extraarticular Clinical Findings of Temporomandibular Dysfunction. Eur. J. Dent. 2011; 5: 354-60.

33. Agerberg G. Maximal mandibular movements in young men and women. Sven Tandlak Tidskr. 1974; 67(2): 81-100.

34. Solberg W. Occlusion-related pathosis and its clinical evaluation. In: Clinical dentistry. New York, NY, USA: Harper \& Row Publishers; 1976: 1-29.

35. Crincoli V, Fatone L, Fanelli M, Rotolo RP, Chialà A, Favia G, Lapadula G. Orofacial Manifestations and Temporomandibular Disorders of Systemic Scleroderma: An Observational Study. Int. J. Mol. Sci. 2016; 17(7): 1189.

36. Liebling MR, Gold RH. Erosions of the temporomandibular joint in systemic lupus erythematosus. Arthritis Rheum. 1981; 24: 948-50.

37. Simões DM, Fava M, Figueiredo MA, Salum FG, Cherubini K. Oral manifestations of lupus erythematosus - report of two cases. Gerodontology. 2013; 30: 303-8.

38. Aceves-Avila FJ, Chávez-López M, Chavira-González JR, Ramos-Remus C. Temporomandibular joint dysfunction in various rheumatic diseases. Reumatismo. 2013; 65: 126-30.

39. Jonsson R, Lindval A, Nyberg G. Temporomandibular joint involvement in systemic lupus erythematosus. Arthritis Rheum. 1983; 26: 1506-10.

40. Kranti K, Seshan H, Juliet J. Discoid lupus erythematosus involving gingiva. J. Indian Soc. Periodontol. 2012; 16: 126-8.

41. Chiewchengchol D, Murphy R, Edwards SW, Beresford MW. Mucocutaneous manifestations in juvenile-onset systemic lupus erythematos us: a review of literature. Pediatr. Rheumatol. Online J. 2015; 13: 1.

42. Bade DM, Lovasko JH, Montana J, Waide FL. Acute closed lock in a patient with lupus erythematosus: case review. J. Craniomandib. Disord. 1992; 6: 208-12. 